\title{
Uncertainty of the coefficient of band-to-band absorption of crystalline silicon at near- infrared wavelengths
}

Carsten Schinke, Karsten Bothe, Peter Christian Peest, Jan Schmidt, and Rolf Brendel

Citation: Appl. Phys. Lett. 104, 081915 (2014);

View online: https://doi.org/10.1063/1.4866916

View Table of Contents: http://aip.scitation.org/toc/apl/104/8

Published by the American Institute of Physics

\section{Articles you may be interested in}

Uncertainty analysis for the coefficient of band-to-band absorption of crystalline silicon

AlP Advances 5, 067168 (2015); 10.1063/1.4923379

Near-infrared free carrier absorption in heavily doped silicon

Journal of Applied Physics 116, 063106 (2014); 10.1063/1.4893176

Empirical determination of the energy band gap narrowing in highly doped $\mathrm{n}^{+}$silicon

Journal of Applied Physics 114, 044508 (2013); 10.1063/1.4816694

Thermo-optic coefficient of silicon at $1550 \mathrm{~nm}$ and cryogenic temperatures

Applied Physics Letters 101, 041905 (2012); 10.1063/1.4738989

Band-gap narrowing in heavily doped silicon: A comparison of optical and electrical data Journal of Applied Physics 63, 425 (1998); 10.1063/1.340257

Empirical determination of the energy band gap narrowing in $\mathrm{p}^{+}$silicon heavily doped with boron Journal of Applied Physics 116, 194505 (2014); 10.1063/1.4902066

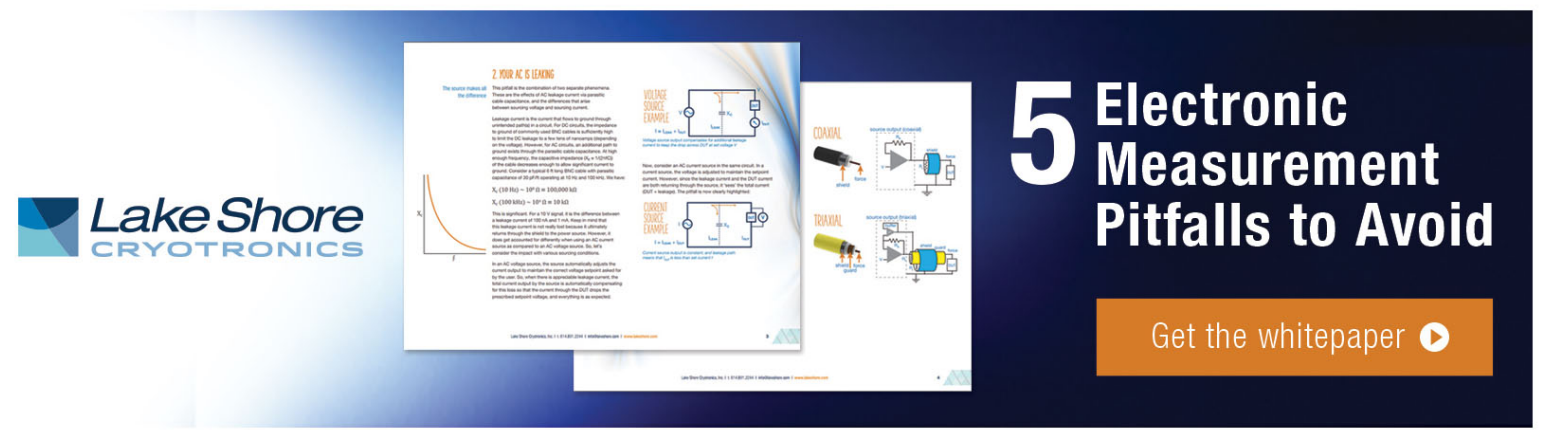




\title{
Uncertainty of the coefficient of band-to-band absorption of crystalline silicon at near-infrared wavelengths
}

\author{
Carsten Schinke, ${ }^{\text {a) }}$ Karsten Bothe, Peter Christian Peest, Jan Schmidt, and Rolf Brendel \\ Institute for Solar Energy Research Hamelin (ISFH), Am Ohrberg 1, D-31860 Emmerthal, Germany
}

(Received 3 February 2014; accepted 13 February 2014; published online 27 February 2014)

\begin{abstract}
We present data of the coefficient of band-to-band absorption of crystalline silicon at $295 \mathrm{~K}$ in the wavelength range from 950 to $1350 \mathrm{~nm}$ and analyze its uncertainty. The data is obtained from measurements of reflectance and transmittance as well as spectrally resolved photoluminescence measurements and spectral response measurements. A rigorous measurement uncertainty analysis based on an extensive characterization of our setups is carried out. We determine relative uncertainties of $4 \%$ at $1000 \mathrm{~nm}$, increasing to $22 \%$ at $1200 \mathrm{~nm}$ and $160 \%$ at $1300 \mathrm{~nm}$, and show that all methods yield comparable results. (C) 2014 AIP Publishing LLC.
\end{abstract}

[http://dx.doi.org/10.1063/1.4866916]

The determination of the coefficient of band-to-band absorption of crystalline silicon is an ongoing research topic since $1955 .{ }^{1-10}$ However, as can be seen from Fig. 1, the datasets from literature show significant deviations in the near-infrared and subbandgap region. ${ }^{9,11,12}$ Critically, it is exactly this wavelength range, which is important for photonical technologies that make use of the transparency of silicon, for imaging applications using silicon detectors as well as for device characterization by luminescence measurements. The datasets shown in Fig. 1 are based on different measurement approaches, namely, measurements of reflectance and transmittance, spectrally resolved luminescence measurements, and spectral response (SR) measurements. Unfortunately, the datasets had been determined at different temperatures ranging from 291 to $300 \mathrm{~K}$ and the authors do not report on substantiated estimates of the uncertainty of their data. Hence, it is unclear whether the deviations are due to sample properties such as temperature or doping concentration, and to which extend they can be explained by the accuracy of the different approaches. Our work therefore aims at comparing the different approaches on the basis of a systematic measurement uncertainty analysis according to the Guide to the Expression of Uncertainty in Measurement (GUM),${ }^{13}$ which is based on an extensive characterization of the measurement setups. This yields data of the absorption coefficient including substantiated estimates of its uncertainty. Deviations between the datasets from literature are discussed with respect to these uncertainties.

Absolute values of the absorption coefficient are usually determined by absorptance measurements on planar samples. The calculation of the absorption coefficient from this data requires knowledge about the reflectance of the sample's surfaces. Alternatively, one might also use a set of reflectance and transmittance measurements, since both, surface reflectance and absorption coefficient, can be calculated directly from this data. However, measuring the absorptance or transmittance in the bandgap and sub-bandgap range is experimentally challenging, since it becomes very small or

\footnotetext{
${ }^{\text {a) }}$ Author to whom correspondence should be addressed. Electronic mail: c.schinke@isfh.de
}

saturates at unity, respectively. One approach for the precise determination of the absorption coefficient in the subbandgap range is the measurement of the luminescence spectrum. This approach makes use of the reciprocity between the absorption and emission of light. ${ }^{14}$ The relation between the absorption coefficient and the luminescence spectrum is defined by the generalized Planck law for luminescence emission. ${ }^{15}$ If either the charge carrier density within the sample is homogenous or the absorption coefficient is very low, the luminescence spectrum is proportional to the absorptance of the sample or the absorption coefficient, respectively. In these cases, the absorption coefficient can be obtained from the luminescence spectrum by scaling the data to a previously determined absolute value of the absorptance or absorption coefficient, respectively. This approach has been demonstrated in Refs. 5 and 8. Due to the scaling, errors and uncertainties of the absolute values propagate into the data from luminescence. The availability of accurate absolute values is therefore critical for the successful application of this method. Another possibility is the

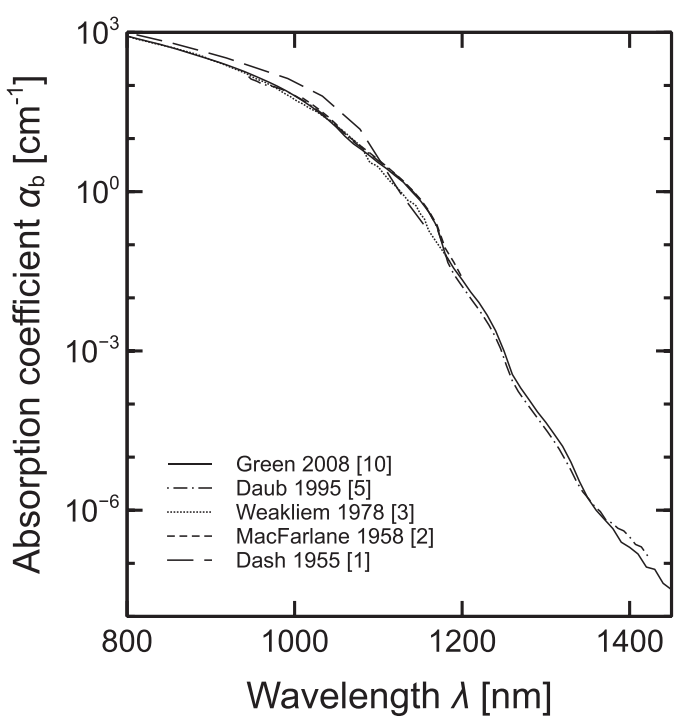

FIG. 1. Comparison of the most widely used datasets of the coefficient of band-to-band absorption of crystalline silicon at room temperature. 
measurement of the SR of silicon solar cells. By an optical reciprocity theorem, ${ }^{16,17}$ the spectral response is related to the luminescence spectrum and thus the same theory applies. This approach has been demonstrated, for instance, in Ref. 6 .

In this work, reflectance/transmittance $(\mathrm{R} / \mathrm{T})$ measurements are carried out on double side polished monocrystalline Czochralski grown $p$-type silicon wafer samples with an area of $3 \times 3 \mathrm{~cm}^{2}$, a resistivity of $6 \Omega \mathrm{cm}$ and thicknesses ranging from 550 to $770 \mu \mathrm{m}$. Moreover, spectrally resolved photoluminescence (PL) measurements are carried out on the same wafers and additionally on double side textured wafers of the same material, which exhibit an enhanced luminescence emission at long wavelengths and thereby allow for measurements with an increased signal-to-noise ratio. For the PL measurements, all samples are passivated on both sides by a $15 \mathrm{~nm}$ thick layer of atomic-layer-deposited $\mathrm{Al}_{2} \mathrm{O}_{3}$ in order to enhance luminescence emission. The passivation layers are characterized by ellipsometry and numerical raytracing simulations in order to ensure that the impact of the passivation layers on the luminescence spectrum is negligible. The sample temperature is $295 \pm 1 \mathrm{~K}$ for all $\mathrm{R} / \mathrm{T}$ measurements and $295 \pm 0.5 \mathrm{~K}$ for all PL measurements carried out in this work. For comparison, SR measurements are performed by the Physikalisch-Technische Bundesanstalt (PTB) in Braunschweig, Germany, on high-efficiency $n$-type back-contact silicon solar cells with a bulk resistivity of $1 \Omega$ $\mathrm{cm}$ at a sample temperature of $298.15 \pm 0.5 \mathrm{~K}$.

$\mathrm{R} / \mathrm{T}$ measurements are performed using a Varian Cary 5000 photospectrometer equipped with an integrating sphere. The system detects radiation either by a photomultiplier or a lead sulfide detector, depending on the wavelength. A reference beam is used to monitor and correct for variations of the illumination intensity over time. The wavelength calibration is automatically performed by the system using a built-in mercury vapor lamp. The calibration with respect to intensity depends on the measurand (reflectance or transmittance) and includes the acquisition of a $100 \%$ baseline and a $0 \%$ baseline. In case of reflectance measurements, the $100 \%$ baseline is defined by the reflectance of a spectralon standard which is primary calibrated at the PTB. The illumination intensity within the system is generally low, and sample heating due to illumination during the measurements is not observed. PL data are acquired using a tec5 CompactSpec 1.7 diode array spectrometer featuring a cooled InGaAs detector. The light is coupled into the device using an optical fiber with a numerical aperture of 0.22 positioned perpendicular above the front surface of the samples at a distance of $3 \mathrm{~cm}$. Luminescence emission is excited by laser light at $808 \mathrm{~nm}$ which homogenously illuminates an area of $5 \times 5 \mathrm{~cm}^{2}$ with an intensity of $100 \mathrm{~mW} / \mathrm{cm}^{2}$. An optical long-pass filter with a cut-off wavelength of $860 \mathrm{~nm}$ circumvents stray light and second order effects caused by the laser light. Spectral stray light caused by the luminescence radiation itself is corrected for. Spectrometer and entrance optics are calibrated with respect to spectral irradiance using a halogen lamp which was primary calibrated at the PTB. The wavelength calibration of the manufacturer is verified by measuring the distinct emission lines of a mercury pencil lamp. Note that a calibration with respect to absolute spectral irradiance is not necessary due to the scaling of the data as described above. The samples are placed on a temperature-controlled black anodized chuck, and a PT1000 temperature sensor is attached to the front surface of the samples during the measurements in order to compensate for sample heating due to illumination. We verify that reflections at the chuck do not affect the shape of the measured luminescence spectrum. A rigorous measurement uncertainty analysis according to the Guide to the Expression of Uncertainty in Measurement ${ }^{13}$ is carried out for all measurements. The analysis includes an extensive characterization of our setups with respect to reproducibility, sample adjustment, measurement amplifier effects, linearity of the detectors, thermal drifts, spectral bandwidth, wavelength accuracy, and spectral stray light. Details about the SR setup at the PTB and the uncertainty analysis for the SR measurements can be found in Refs. 18 and 19 .

The absorption coefficient data and its expanded uncertainty for a coverage probability of $95 \%$ (corresponding to a coverage factor $k=2$, see Ref. 13) as determined from our measurements are given in Table I and are shown in Fig. 2

TABLE I. Absorption coefficient of crystalline silicon at $295 \mathrm{~K}$.

\begin{tabular}{|c|c|c|}
\hline$\lambda(\mathrm{nm})$ & $\alpha_{\mathrm{b}}(1 / \mathrm{cm})$ & $U\left(\alpha_{\mathrm{b}}\right) / \alpha_{\mathrm{b}}(\mathrm{k}=2)(\%)$ \\
\hline 950 & $1.499 \times 10^{2}$ & 7.8 \\
\hline 960 & $1.293 \times 10^{2}$ & 3.4 \\
\hline 970 & $1.098 \times 10^{2}$ & 1.9 \\
\hline 980 & $9.239 \times 10^{1}$ & 1.5 \\
\hline 990 & $7.500 \times 10^{1}$ & 5.6 \\
\hline 1000 & $6.208 \times 10^{1}$ & 3.8 \\
\hline 1010 & $4.876 \times 10^{1}$ & 2.2 \\
\hline 1020 & $3.870 \times 10^{1}$ & 1.7 \\
\hline 1030 & $2.914 \times 10^{1}$ & 1.5 \\
\hline 1040 & $2.151 \times 10^{1}$ & 1.5 \\
\hline 1050 & $1.541 \times 10^{1}$ & 1.6 \\
\hline 1060 & $1.086 \times 10^{1}$ & 1.8 \\
\hline 1070 & $7.796 \times 10^{0}$ & 2.0 \\
\hline 1080 & $5.960 \times 10^{0}$ & 2.3 \\
\hline 1090 & $4.490 \times 10^{0}$ & 2.6 \\
\hline 1100 & $3.381 \times 10^{0}$ & 3.3 \\
\hline 1110 & $2.527 \times 10^{0}$ & 3.9 \\
\hline 1120 & $1.864 \times 10^{0}$ & 5.1 \\
\hline 1130 & $1.339 \times 10^{0}$ & 5.8 \\
\hline 1140 & $9.229 \times 10^{-1}$ & 5.6 \\
\hline 1150 & $5.887 \times 10^{-1}$ & 5.6 \\
\hline 1160 & $3.445 \times 10^{-1}$ & 5.7 \\
\hline 1170 & $1.623 \times 10^{-1}$ & 7.0 \\
\hline 1180 & $4.093 \times 10^{-2}$ & 14 \\
\hline 1190 & $2.079 \times 10^{-2}$ & 20 \\
\hline 1200 & $1.227 \times 10^{-2}$ & 22 \\
\hline 1210 & $7.363 \times 10^{-3}$ & 23 \\
\hline 1220 & $4.404 \times 10^{-3}$ & 23 \\
\hline 1230 & $2.447 \times 10^{-3}$ & 24 \\
\hline 1240 & $1.207 \times 10^{-3}$ & 27 \\
\hline 1250 & $4.357 \times 10^{-4}$ & 40 \\
\hline 1260 & $1.772 \times 10^{-4}$ & 66 \\
\hline 1270 & $1.030 \times 10^{-4}$ & 84 \\
\hline 1280 & $6.278 \times 10^{-5}$ & 100 \\
\hline 1290 & $3.824 \times 10^{-5}$ & 130 \\
\hline 1300 & $2.401 \times 10^{-5}$ & 160 \\
\hline 1310 & $1.357 \times 10^{-5}$ & 210 \\
\hline 1320 & $7.193 \times 10^{-6}$ & 310 \\
\hline 1330 & $3.235 \times 10^{-6}$ & 550 \\
\hline 1340 & $1.229 \times 10^{-6}$ & 1200 \\
\hline 1350 & $6.274 \times 10^{-7}$ & 1800 \\
\hline
\end{tabular}



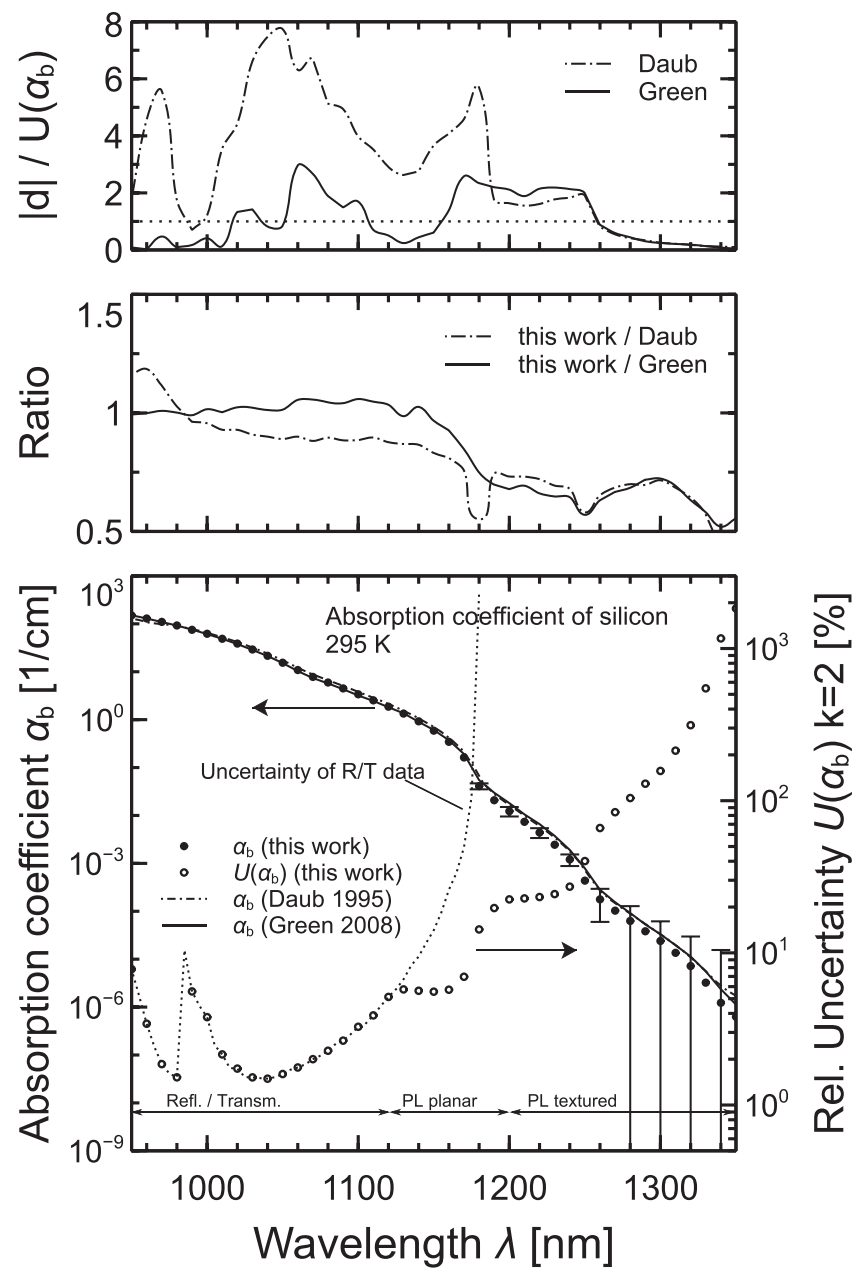

FIG. 2. Band absorption coefficient $\alpha_{\mathrm{b}}$ of crystalline silicon at $295 \mathrm{~K}$ as determined from the measurements carried out in this study (bottom graph). For comparison, literature data by Daub $^{5}$ and Green ${ }^{10}$ are visualized by the lines. The dotted line visualizes the uncertainty of $\mathrm{R} / \mathrm{T}$ data. The middle graph shows the ratio of our to the literature data. The top graph compares the deviation to the estimated uncertainty of our data.

(bottom graph). The expanded uncertainty is calculated from the standard uncertainty (corresponding to a coverage probability of $68 \%$, i.e., $k=1$ ) by multiplication with 2 . For a definition of the coverage factor, please also refer to Fig. 4. Additionally, the datasets by Daub ${ }^{5}$ and Green ${ }^{10}$ are plotted since they are also specified for $295 \mathrm{~K}$. Below $1120 \mathrm{~nm}$, our data are deduced from $\mathrm{R} / \mathrm{T}$ measurements. The wavelength range from 1120 to $1200 \mathrm{~nm}$ is covered by $\mathrm{PL}$ data of a polished sample, which is scaled to the data from $\mathrm{R} / \mathrm{T}$ in the wavelength range between 1100 and $1150 \mathrm{~nm}$. This range is chosen such that the uncertainty of the scaling factor is minimized. Using data at several wavelengths for scaling reduces the uncertainty of the scaling factor compared to using only one wavelength for scaling. For wavelengths above $1200 \mathrm{~nm}$, PL data from a textured sample are used and scaled to the data from PL of the planar sample in the wavelength range between 1200 and $1250 \mathrm{~nm}$. The middle graph of Fig. 2 shows the ratio of our data to that of Green and Daub. At wavelengths below the bandgap $(1150 \mathrm{~nm})$, the ratio of our data to that of Green is around unity. Compared to the values of Daub, our data are smaller by a factor of around 0.9 . Above $1150 \mathrm{~nm}$, our values are smaller by a factor of approximately 0.6 . In the top graph of Fig. 2, the absolute value of the deviation $d=\alpha_{\mathrm{b}}$ (this work) $-\alpha_{\mathrm{b}}$ (lit.) between our and literature data is compared to the uncertainty $U$ of our data (specified for $k=2$ ). Values below unity mean that the deviation is smaller than the estimated uncertainty of our data (see Fig. 4). The plot shows that the deviations can only partly be explained by the uncertainty of our data (in the regions where $|d| / U \leq 1)$. This points towards systematic effects due to, e.g., sample temperature or stray light. Note that the uncertainty of the literature data is unknown and thus not taken into account for this analysis. The relative uncertainty of our data increases from $4 \%$ at $1100 \mathrm{~nm}$ to $22 \%$ at $1200 \mathrm{~nm}$ and $160 \%$ at $1300 \mathrm{~nm}$. The increase is mainly due to the strongly decreasing signal-to-noise ratio and spectral stray light. Future work will aim at the reduction of uncertainty in this region. The uncertainty of the absorption coefficient deduced from $\mathrm{R} / \mathrm{T}$ measurements increases steeply to $100 \%$ rel. at $1175 \mathrm{~nm}$ (see dotted line in Fig. 2), showing that scaling of PL or SR data to R/T data at wavelengths of $1180 \mathrm{~nm}$ or above as demonstrated, e.g., in Ref. 6, may be subject to large uncertainties, which propagate into the data at longer wavelengths. This might also provide an insight into the deviation between our data and the data of Green, which is partly based on the data of Ref. 6 .

Figure 3 compares the absorption coefficient as deduced from PL to that deduced from SR measurements carried out in the wavelength range from 900 to $1300 \mathrm{~nm}$. The SR data are scaled to the absorption coefficient from PL between 1200 and $1250 \mathrm{~nm}$. The top graph shows the ratio of PL to SR data as well as the deviation compared to the uncertainty of the PL data. Values of $|d| / U(k=2) \leq 0.5$ for all wavelengths show that the SR data agree with the PL data within one standard deviation of the latter (see Fig. 4), which means that both methods yield comparable results. As the SR data are measured at higher temperature and possible systematic deviations due to the highly doped layers within the solar cell have not been investigated yet, the SR data are only

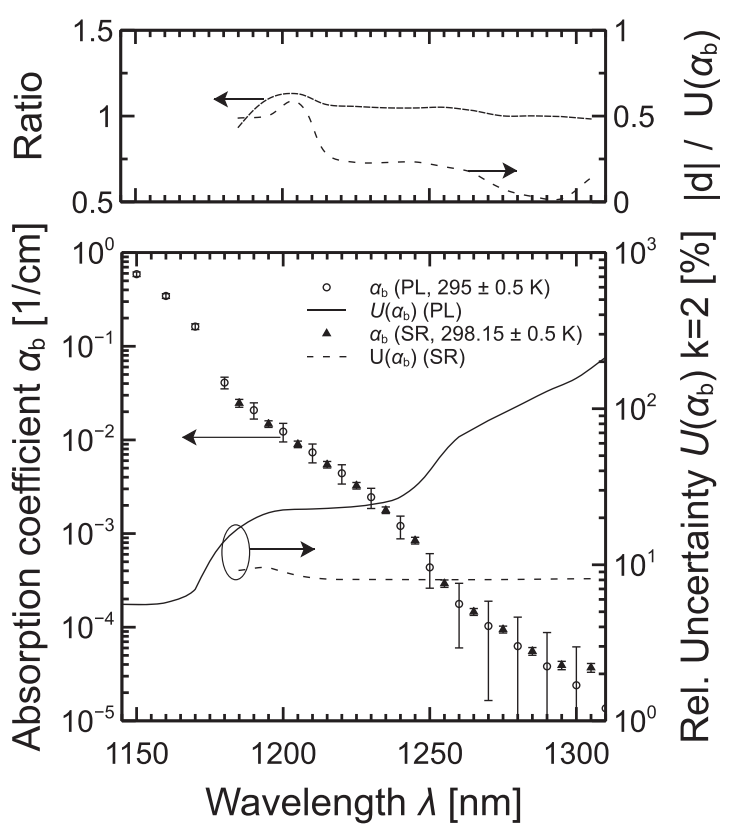

FIG. 3. Comparison of the coefficient of band-to-band absorption as deduced from photoluminescence measurements at ISFH and spectral response measurements carried out by the PTB. 


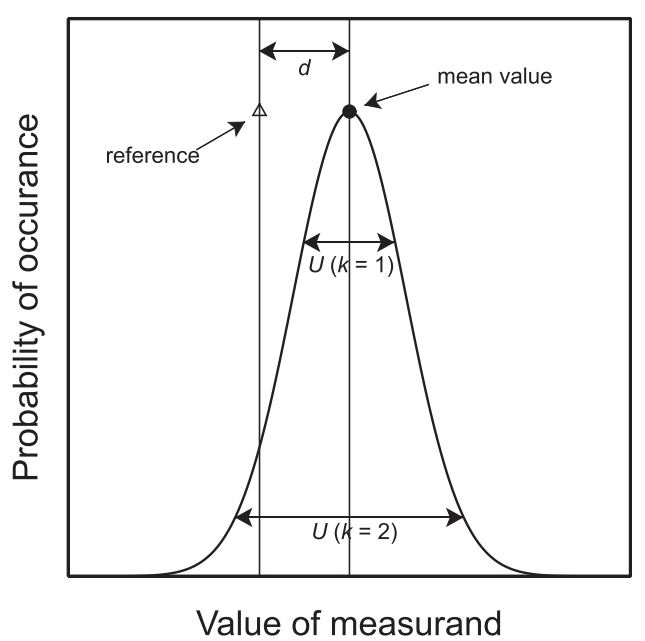

FIG. 4. Visualization of mean value and uncertainty $U$ for a normally distributed measurand and the deviation $d$ between the mean value of the measurand and a reference value.

used for comparison and not given in Table I. The uncertainty of the SR data at $295 \mathrm{~K}$ is comparable. Correcting for temperature using the temperature coefficients given in Ref. 10 before scaling would shift down the SR data around $1300 \mathrm{~nm}$ by about $5 \%$ rel., thus leading to even better agreement between PL and SR. The accurate determination of the absorption coefficient from SR measurements will be subject of future work.

In summary, it is shown that the combination of reflectance/transmittance and spectrally resolved photoluminescence measurements allows the absorption coefficient of crystalline silicon to be determined with relative uncertainties below $6 \%$ up to $1150 \mathrm{~nm}$, increasing to $160 \%$ at $1300 \mathrm{~nm}$. Our data are confirmed by SR measurements carried out at the PTB. Reasonable agreement between our and literature data is found. The uncertainty analysis shows that scaling of PL or SR data to $\mathrm{R} / \mathrm{T}$ data at wavelengths of
$1180 \mathrm{~nm}$ or above as demonstrated in the literature can be subject to large uncertainties.

The authors would like to thank S. Winter and I. Kröger from the Physikalisch-Technische Bundesanstalt (PTB) in Braunschweig, Germany, for carrying out spectral response measurements. The authors would also like to thank B. Falster from MEMC and E. Daub from Siltronic AG for providing silicon wafer samples. The help of M. Offer, D. Hinken, P. P. Altermatt, C. Marquardt, and A. Merkle from ISFH is also gratefully acknowledged. Funding was provided by the scholarship program of the German Federal Environmental Foundation (Deutsche Bundesstiftung Umwelt) and the State of Lower Saxony.

${ }^{1}$ W. C. Dash and R. Newman, Phys. Rev. 99, 1151 (1955).

${ }^{2}$ G. G. Macfarlane, T. P. McLean, J. E. Quarrington, and V. Roberts, Phys. Rev. 111, 1245 (1958).

${ }^{3}$ H. A. Weakliem and D. Redfield, J. Appl. Phys. 50, 1491 (1979).

${ }^{4}$ G. E. Jellison, Opt. Mater. 1, 41 (1992).

${ }^{5}$ E. Daub and P. Würfel, Phys. Rev. Lett. 74, 1020 (1995).

${ }^{6}$ M. J. Keevers and M. A. Green, Appl. Phys. Lett. 66, 174 (1995).

${ }^{7}$ C. M. Herzinger, B. Johs, W. A. McGahan, J. A. Woollam, and W. Paulson, J. Appl. Phys. 83, 3323 (1998).

${ }^{8}$ T. Trupke, E. Daub, and P. Würfel, Sol. Energy Mater. Sol. Cells 53, 103 (1998).

${ }^{9}$ J. Geist, Handbook of Optical Constants of Solids III (Academic Press, New York, 1998).

${ }^{10}$ M. A. Green, Sol. Energy Mater. Sol. Cells 92, 1305 (2008).

${ }^{11}$ C. Schinke, D. Hinken, J. Schmidt, K. Bothe, and R. Brendel, IEEE J. Photovoltaics 3, 1038 (2013).

${ }^{12}$ B. Mitchell, M. K. Juhl, M. A. Green, and T. Trupke, IEEE J. Photovoltaics 3, 962 (2013).

${ }^{13}$ Joint Committee for Guides in Metrology, Guide to the Expression of Uncertainty in Measurement (BIPM, Paris, 2008).

${ }^{14}$ G. Kirchhoff, Ann. Phys. 185, 275 (1860).

${ }^{15} \mathrm{P}$. Würfel, Appl. Phys. A 60, 67 (1995).

${ }^{16}$ U. Rau, Phys. Rev. B 76, 085303 (2007).

${ }^{17}$ U. Rau, IEEE J. Photovoltaics 2, 169 (2012).

${ }^{18} \mathrm{~S}$. Winter, T. Wittchen, and J. Metzdorf, in Proceedings of the 16th European Photovoltaic Solar Energy Conference and Exhibition (Glasgow, UK, 2000), p. 2198.

${ }^{19} \mathrm{~S}$. Winter and A. Sperling, in Proceedings of the 2nd Expert Symposium on Measurement Uncertainty (Braunschweig, Germany, 2006), p. 139. 